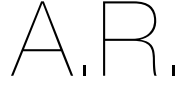

ARTIGO DE REVISÃO

1 Instituto Politécnico de Coimbra, Escola Superior de Tecnologia da Saúde de Coimbra,

Rua 5 de Outubro,

3040-655 Coimbra,

Portugal

2 Laboratório de

Investigação Aplicada em

Saúde (LabinSaúde),

Rua 5 de Outubro -

S. Martinho Bispo,

Apartado 7006,

3046-854 Coimbra,

Portugal

"Endereço para correspondência:

Diogo V Martinho

Instituto Politécnico de Coimbra,

Escola Superior de Tecnologia

Saúde de Coimbra,

da Saúde de Coimb

(2040-655 Coimbra, Portuga

dvmartinh092@hotmail.com

Histórico do artigo:

Recebido a 15 de julho de 202 Aceite a 17 de dezembro de 202

\section{O CONSUMO DE HIDRATOS DE CARBONO EM FUTEBOLISTAS MASCULINOS: REVI- SÃO SISTEMÁTICA E META-ANÁLISE}

\author{
CARBOHYDRATE INTAKE AMONG MALE SOCCER PLAYERS: \\ A SYSTEMATIC REVIEW AND META-ANALYSIS
}

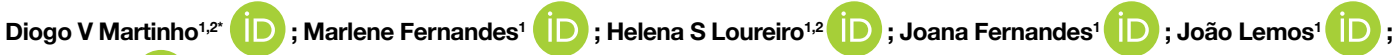
Ana Faria ${ }^{1,2}$ iD

RESUMO

INTRODUÇÃo: As diretrizes relativas ao consumo de hidratos de carbono têm sido extensivamente discutidas. Apesar deste macronutriente ser reconhecidamente determinante no rendimento desportivo, escassas revisões sistemáticas e meta-análises foram conduzidas para avaliar a ingestão de hidratos de carbono em futebolistas masculinos.

OBJETIVOS: O objetivo desta revisão sistemática, com meta-análise, foi avaliar a ingestão de hidratos de carbono em diferentes períodos da época competitiva. Adicionalmente, foi examinado o consumo de hidratos de carbono por grupo posicional.

METODOLOGIA: As bases de dados utilizadas foram PubMed, SPORTDiscus e Web of Science Core Collection. Os estudos incluídos consideraram os seguintes critérios de elegibilidade: jogadores de futebol masculino, idade superior a 18 anos e consumo de hidratos de carbono expresso em g.kg-1.dia-1

RESULTADOS: Inicialmente, foram identificados 1992 artigos. Destes, 11 foram considerados elegíveis para a revisão sistemática e 10 foram incluídos na meta-análise. Os resultados demonstraram que os futebolistas masculinos, consomem em média, 4,3 g.kg$\mathrm{dia}^{-1}$ e 5,0 g. $\mathrm{kg}^{-1}$. dia-1 nos períodos competitivos e preparatórios (pré-época), respetivamente. Não foi encontrado qualquer estudo que reportasse o consumo de hidratos de carbono durante a off-season. Apenas três estudos examinaram a variação do consumo de hidratos de carbono por grupo posicional. Embora as amostras sejam limitadas, os guarda-redes parecem ingerir uma menor quantidade de hidratos de carbono comparativamente a atletas de outras posições em campo.

CONCLUSÕES: Considerando as limitações associadas às metodologias utilizadas para aferir o consumo de hidratos de carbono em atletas, a presente revisão sistemática realça a necessidade de desenvolver estratégias nutricionais para o cumprimento das diretrizes deste macronutriente.

PALAVRAS-CHAVE

Competição, Energia, Glicogénio, Macronutrientes, Periodização

ABSTRACT

INTRODUCTION: Guidelines for carbohydrate $(\mathrm{CHO})$ intake have been systematically updated. Although $\mathrm{CHO}$ are determinant to soccer performance, few systematic reviews or meta-analyses described the $\mathrm{CHO}$ ingestion among male soccer players. OBJECTIVES: This systematic-review, with meta-analyses, examined the $\mathrm{CHO}$ intake in male soccer players according to the competitive schedule (preparatory season, season and off-season). Additionally, the variability of $\mathrm{CHO}$ intake considering playing position was described.

METHODOLOGY: Three databases were consulted: PubMed, SPORTDiscus and Web of Science Core Collection. Studies were included according to the following eligibility criteria: (i) male soccer players, (ii) age $\geq 18.0$ years, (iii) $\mathrm{CHO}$ intake expressed in $\mathrm{g} \mathrm{kg}^{-1}$.day $\mathrm{d}^{-1}$. RESULTS: Initially, 1992 manuscripts were identified. Of those, 11 manuscripts were considered eligible for the systematic review and 10 manuscripts were included in the meta-analyses. The results of the current meta-analysis showed that male soccer players consumed, on average, $4.3 \mathrm{~g} . \mathrm{kg}^{-1}$. day ${ }^{-1}$ and $5.0 \mathrm{~g} \mathrm{~kg}^{-1}$. day ${ }^{-1}$ of $\mathrm{CHO}$ during the season and in the preparatory period, respectively. Surprisingly, no studies reported $\mathrm{CHO}$ intake during off-season. Only three studies examined $\mathrm{CHO}$ ingestion by playing position. Although samples were limited, goalkeepers seemed to ingest less $\mathrm{CHO}$ than outfielders.

CONCLUSIONS: Considering the limitations associated with $\mathrm{CHO}$ intake reporting, nutritional strategies to confirm that athletes meet the intake of this macronutrient are needed.

KEYWORDS

In-season, Energy, Glycogen, Macronutrients, Periodization 


\section{INTRODUÇÃO}

Os hidratos de carbonos no futebol revelam-se fundamentais na produção de energia e, por isso, são determinantes no rendimento desportivo. Em 31 jogadores dos Países Baixos, o glicogénio muscular diminuiu 46\% após a realização de jogos não-oficiais (1). Para comprovar o impacto dos hidratos de carbono no rendimento, os participantes, realizaram um protocolo intermitente antes e depois dos jogos (1). O tempo médio de realização do teste decresceu $2,8 \%$ comparativamente aos valores iniciais. Este estudo veio reforçar resultados de pesquisas precedentes, em que a depleção de $40-90 \%$ do glicogénio muscular durante a competição afetaram negativamente o rendimento em esforços maximais de curta duração $(2,3)$. Adicionalmente, um estudo transversal dos anos 90 examinou, em 6 jogadores adultos, a associação entre a concentração de glicogénio e os esforços predominantes em 90 minutos de jogo (4). Curiosamente, o tempo despendido em esforços maximais esteve substancialmente associado à concentração de glicogénio muscular avaliada antes da competição. Por outras palavras, os participantes que recorreram a uma dieta predominante em hidratos de carbono (65\% do consumo energético total), 48 horas antes da competição realizaram ações de alta intensidade durante mais tempo.

Durante as últimas décadas, as diretrizes para o consumo de hidratos de carbono em futebolistas masculinos foram extensivamente documentadas, debatidas e atualizadas (5-7). Inicialmente, 5-7 g. $\mathrm{kg}^{-1}$. $\mathrm{dia}^{-1}$ de hidratos de carbono foram recomendadas para períodos de treino moderados (6). Para suportar o período competitivo, caracterizado por sessões de treino intensas, e a necessidade de abastecer para os momentos competitivos, foram sugeridas $7-12 \mathrm{~g} \cdot \mathrm{kg}^{-1}$. dia ${ }^{-1}$ (6) Recentemente, as recomendações de hidratos de carbono foram revistas por um grupo de peritos: para o período preparatório 4-8 g. $\mathrm{kg}^{-1}$. $\mathrm{dia}^{-1} ; 3-8 \mathrm{~g} \mathrm{~kg}^{-1}$. dia-1 foram propostas para a fase competitiva, permitindo aos atletas suportar treinos e jogos, assim como repor as reservas de glicogénio; $<4 \mathrm{~g} \mathrm{~kg}^{-1}$. dia ${ }^{-1}$ foram sugeridas durante a off-season para a otimização da composição corporal (7). Assim, um consumo apropriado de hidratos de carbono, considerando a variabilidade de carga imposta durante a época competitiva, tem um impacto positivo nas adaptações ao treino e competição em futebolistas $(5,7)$.

A variação do consumo de hidratos de carbono por grupo, em esforços maximais avaliados através da monitorização da carga de treino e de avaliações laboratoriais, foi documentada em futebolistas de elite (8, 9). Por exemplo, com recurso à tecnologia global positioning system (GPS), foi reportado que os médios apresentavam valores superiores de velocidade média comparativamente aos avançados, e que os defesas percorriam uma menor distância em esforços maximais, em contraste com os alas, médios e avançados (8). Além disso, os avançados evidenciaram melhores desempenhos em protocolos de terreno do que os guarda-redes, defesas e médios (9). Em paralelo, o gasto energético total também variou por grupo posicional. Recentemente, dois estudos examinaram o dispêndio energético total, com recurso a água duplamente marcada, em futebolistas de elite $(10,11)$. Apesar dos estudos anteriormente mencionados serem limitados no tamanho amostral, diferenças assinaláveis foram evidentes entre os guarda-redes e as restantes posições no campo. O dispêndio energético em seis jogadores (defesas, médios e avançados) foi, em média, de 3566 kcal por dia (11), enquanto o guarda-redes dispendeu $2894 \mathrm{kcal}$ (10). Consequentemente, as diretrizes de hidratos de carbono não devem ser generalizáveis para os diferentes grupos posicionais, particularmente, para guarda-redes que gastam menos energia por dia comparativamente às restantes posições no campo.
Dada a relação entre o glicogénio muscular, o consumo de hidratos de carbono e o impacto no rendimento desportivo, as recomendações de hidratos de carbono devem ser prescritas de acordo com a periodização da época $(7,12)$. O primeiro objetivo deste estudo foi examinar se o consumo de hidratos de carbono em futebolistas obedece às diretrizes recomendadas. Adicionalmente, foi também testada a variação no consumo de hidratos de carbono por grupo posicional.

\section{METODOLOGIA}

\section{Protocolo}

O presente estudo foi realizado considerando as recomendações propostas pelo Preferred Reporting Items for Systematic Reviews and Meta-Analyses (13).

\section{Elegibilidade e Critérios de Inclusão}

As pesquisas científicas arbitradas por pares foram selecionadas para a presente revisão. Os manuscritos elegíveis obedeceram aos seguintes critérios de inclusão: (i) amostra com jogadores de futebol masculino; (ii) idade superior a 18 anos; (iii) o consumo de hidratos de carbono ser expresso em gramas por quilograma de massa corporal.

\section{Pesquisa}

A estratégia de pesquisa foi realizada no dia 13 de abril de 2021 em três base de dados, a saber: PubMed, SPORTDiscus e Web of Science Core Collection. Os termos de pesquisa usados envolveram a combinação das seguintes palavras: "carbohydrate ingestion", " $\mathrm{CHO}$ intake", "CHO ingestion", "carbohydrate need", "CHO need", "adult male soccer", "professional male soccer". Subsequentemente, os manuscritos foram selecionados com recurso ao software CADIMA (14). Tendo em conta critérios de elegibilidade, os manuscritos foram primeiramente considerados por título e abstract, e numa segunda fase pelo texto completo. Este processo foi realizado por dois observadores.

\section{Itens da Pesquisa}

Os participantes do sexo masculino estiveram envolvidos na prática organizada de futebol. O consumo de hidratos de carbono foi expresso em g. $\mathrm{kg}^{-1} \cdot \mathrm{dia}^{-1}$ e comparado com as recomendações definidas para futebolistas (5-7)

\section{Risco de Enviesamento dos Estudos}

O risco de enviesamento dos estudos foi avaliado através de uma ferramenta desenvolvida para estudos observacionais (15). Esta é constituída por oito questões com resposta categórica: yes, no, unclear, not applicable. As questões referem-se à amostra envolvida, à qualidade dos dados, às co-variáveis que devem ser consideradas e finalmente, aos procedimentos estatísticos usados.

\section{Síntese de Resultados}

Os estudos foram agrupados de acordo com as recomendações de hidratos de carbono definidas: para a pré-epoca e durante o período competitivo. Surpreendentemente, nenhum estudo examinou o consumo de hidratos de carbono na off-season. Os estudos que apresentaram os resultados separadamente para dias de treino e de jogo foram incluídos na mesma meta-análise, uma vez que se trata do período competitivo. Surpreendentemente, a média e o desvio padrão referentes ao período de descanso, durante a semana competitiva, não foram incluídas na análise. A heterogeneidade foi examinada pela estatística I e interpretada da seguinte forma (16): I < 25\% (baixa); $25 \% \leq 1<75 \%$ (moderado); $\mid \geq 75 \%$ (alta). A meta-análise foi conduzida 
no Meta-Analyst software (version beta 3.13) e o nível de significância foi definido $a<0,05$. Apenas dois artigos discriminaram a variação por grupo posicional, adotando diferentes categorias, razão pela a qual não foi realizada a meta-análise. Finalmente, um estudo-caso que avaliou o consumo energético de um guarda-redes durante uma semana também foi incluído na variação por grupo posicional.

\section{RESULTADOS}

\section{Identificação e Seleção dos Estudos}

A pesquisa nas bases de dados identificou 1992 títulos. Após remover os duplicados ( $n=132), 1860$ artigos foram rastreados através do título e do abstract. Destes, 1834 registos foram excluídos. O texto dos 26 artigos elegíveis para a seleção foi cuidadosamente examinado, sendo 15 excluídos pelas seguintes razões: não era reportado o consumo de hidratos de carbono, o consumo de hidratos de carbono não era expresso em g. $\mathrm{kg}^{-1}$. dia-1 a amostra envolveu jovens futebolistas e finalmente, quando a mesma amostra foi objeto de estudo em estudos diferentes (Figura 1). Dos 11 estudos elegíveis, 10 foram incluídos na meta-análise.

As características dos 11 estudos envolvidos nesta revisão sistemática são apresentadas na Tabela 1. A maioria dos estudos são observacionais e procuram avaliar o consumo de hidratos de carbono em amostras de futebolistas específicas de um determinado país: Espanha (17, 18), Japão (19), Austrália (20, 21), Países Baixos $(22,23)$, Inglaterra $(10,11)$, Brasil (24) e Polónia (25). O nível dos atletas variou entre futebolistas que treinavam pelo menos quatro vezes por semana a um nível profissional. Com exceção do estudo que envolveu futebolistas japoneses (19), todos usaram o recordatório das 24 horas anteriores ou o diário de frequência alimentar para estimar o consumo alimentar, ainda que tenham recorrido a diferentes períodos temporais. Como referido anteriormente, um caso-estudo que reportou o consumo de hidratos de carbono num guarda-redes durante uma semana (10), foi excluído da meta-análise, mas considerado para examinar a variabilidade no consumo de hidratos de carbono por grupo posicional.

\section{Qualidade Metodológica dos Estudos}

A ferramenta utilizada para analisar o risco de enviesamento de cada estudo permitiu aferir que os critérios de inclusão na maioria das amostras não são discriminados. O estudo que envolveu a amostra de futebolistas japonenses (19) recorreu ao questionário de frequência alimentar adaptado para aquele país. No entanto, apesar de este ter sido previamente validado, não é considerado o procedimento apropriado para avaliar o consumo alimentar em atletas.

\section{Consumo de Hidratos de Carbono Durante o Período Competitivo}

Seis estudos foram incluídos na meta-análise, sendo a média do consumo de hidratos de carbono para o total de futebolistas considerados ( $\mathrm{n}=205)$ de 4,3 g. $\mathrm{kg}^{-1} \cdot \mathrm{dia}^{-1}\left(95 \% \mathrm{IC}: 3,9 \mathrm{~g} \cdot \mathrm{kg}^{-1} \cdot \mathrm{dia}^{-1}\right.$ a 4,6 $\left.\mathrm{g} \cdot \mathrm{kg}^{-1} \cdot \mathrm{dia}^{-1}\right)$, durante o período competitivo. O valor de heterogeneidade foi elevado (l = 91\%; p<0,001) - Figura 2.

\section{Figura 1}

Diagrama PRISMA que representa o processo de seleção dos estudos incluídos na revisão sistemática e na meta-análise
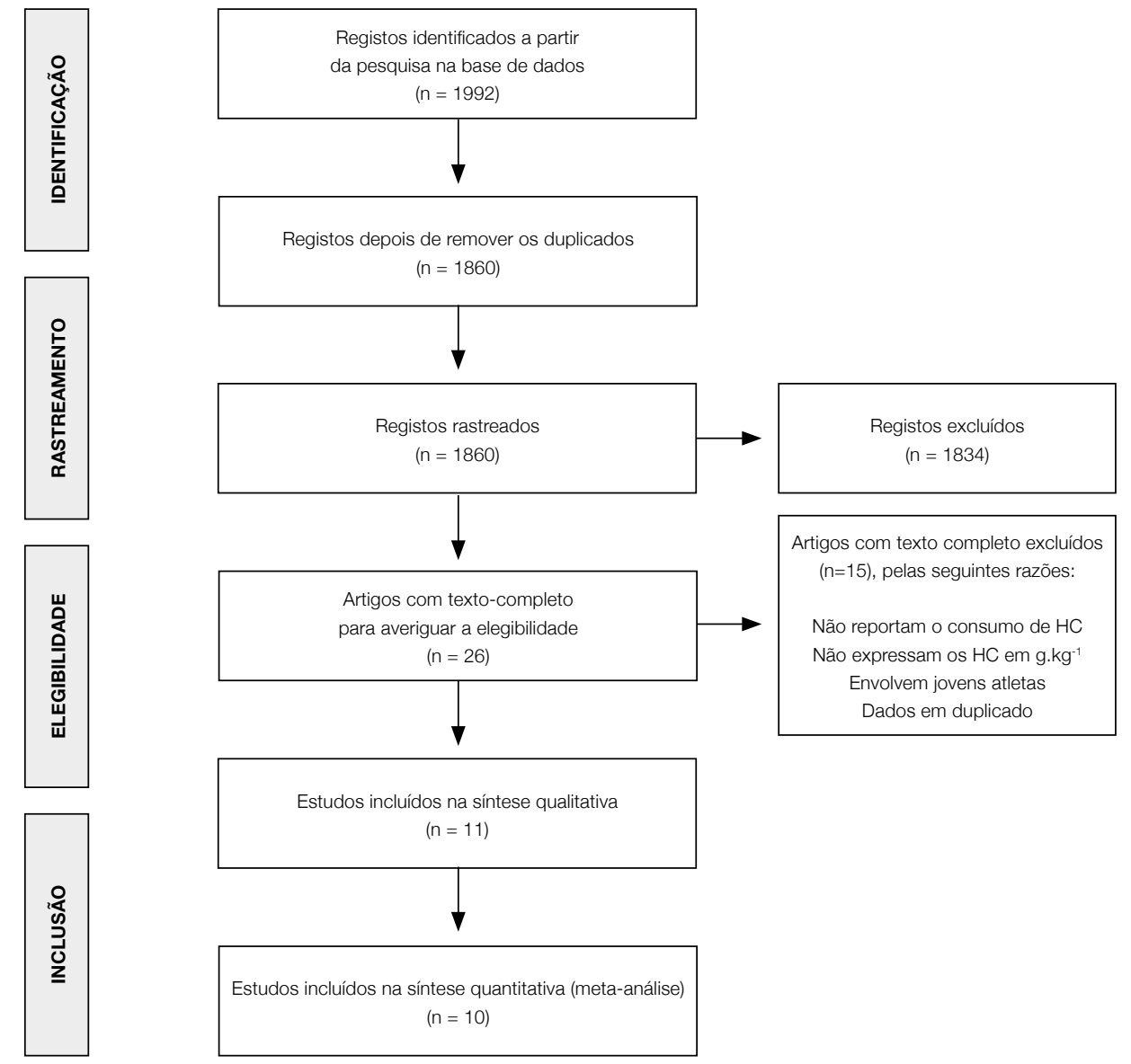
Tabela 1

Características dos estudos incluídos e metodologia usada para a estimativa do consumo de hidratos de carbono

\begin{tabular}{|c|c|c|c|c|c|c|c|c|}
\hline ESTUDO & PAís & $\begin{array}{l}\text { NÍVEL } \\
\text { COMPETITIVO }\end{array}$ & $\begin{array}{l}\text { NÚMERO DE } \\
\text { PARTICIPANTES }\end{array}$ & $\begin{array}{l}\text { PERÍODO DA } \\
\text { ÉPOCA }\end{array}$ & IDADE & $\begin{array}{l}\text { MÉDIA } \\
\text { CONSUMO HC }\end{array}$ & $\begin{array}{l}\text { DESVIO PADRÃO } \\
\text { CONSUMO HC }\end{array}$ & $\begin{array}{l}\text { METODOLOGIA } \\
\text { PARA A ESTIMATIVA } \\
\text { DO CONSUMO } \\
\text { ALIMENTAR }\end{array}$ \\
\hline Ruiz et al., 2005 & Espanha & treino regular & 24 & durante a época & 20,9 & 4,6 & 0,2 & $\begin{array}{l}\text { diário alimentar } \\
3 \text { dias }\end{array}$ \\
\hline Noda et al., 2009 & Japão & treino regular & 31 & pré-epoca & 19,0 & 6,9 & 2,4 & $\begin{array}{l}\text { questionário } \\
\text { frequência alimentar }\end{array}$ \\
\hline $\begin{array}{l}\text { Reñon \& Collado, } \\
2013\end{array}$ & Espanha & semi-profissional & 21 & dia de jogo & 25,9 & 4,2 & 1,3 & $\begin{array}{l}\text { recordatório das } 24 \\
\text { horas anteriores }\end{array}$ \\
\hline $\begin{array}{l}\text { Reñon \& Collado, } \\
2013\end{array}$ & Espanha & semi-profissional & 21 & treino & 25,9 & 3,6 & 0,8 & \\
\hline Devlin et al., 2016 & Austrália & Elite & 18 & pré-epoca & 27 & 2,9 & 1,1 & $\begin{array}{l}\text { recordatório das } 24 \\
\text { horas anteriores }\end{array}$ \\
\hline $\begin{array}{l}\text { Bettonviel et al., } \\
2016\end{array}$ & Países Baixos & profissional & 14 & durante a época & 22,8 & 4.7 & 0,7 & $\begin{array}{l}\text { recordatório das } 24 \\
\text { horas anteriores }\end{array}$ \\
\hline $\begin{array}{l}\text { Andrews et al., } \\
2016\end{array}$ & Austrália & profissional & $10 a$ & durante a época & $21(18-33)$ & 3.9 & 1,8 & diário alimentar 3 dias \\
\hline $\begin{array}{l}\text { Andrews et al., } \\
2016\end{array}$ & Austrália & semi-profissional & $31 b$ & durante a época & $22(18-37)$ & 3,5 & 0,8 & \\
\hline $\begin{array}{l}\text { Anderson et al., } \\
2017\end{array}$ & Inglaterra & profissional & 6 & dia de jogo & 27 & 6,4 & 2,2 & $\begin{array}{l}\text { diário alimentar } 7 \text { dias } \\
\text { e recordatório das } 24 \\
\text { horas anteriores }\end{array}$ \\
\hline $\begin{array}{l}\text { Anderson et al., } \\
2017\end{array}$ & Inglaterra & profissional & 6 & treino & 27 & 4,2 & 1,4 & $\begin{array}{l}\text { diário alimentar } 7 \text { dias } \\
\text { e recordatório das } 24 \\
\text { horas anteriores }\end{array}$ \\
\hline Raizel et al., 2017 & Brasil & profissional & 19 & pré-epoca & 20,71 & 5,4 & 1,9 & diário alimentar 3 dias \\
\hline $\begin{array}{l}\text { Anderson et al., } \\
2018^{\star}\end{array}$ & Inglaterra & profissional & 1 & durante a época & 27 & 2,6 & 0,6 & $\begin{array}{l}\text { recordatório das } 24 \\
\text { horas anteriores diário } \\
\text { alimentar } 7 \text { dias }\end{array}$ \\
\hline $\begin{array}{l}\text { Brinkmans et al., } \\
2019\end{array}$ & Países Baixos & profissional & 41 & durante a época & 23 & 4,0 & 1,2 & $\begin{array}{l}\text { recordatório das } 24 \\
\text { horas anteriores }\end{array}$ \\
\hline $\begin{array}{l}\text { Kziazek et al., } \\
2020\end{array}$ & Polónia & profissional & 26 & pré-epoca & 27 & 5,1 & 1,2 & diário alimentar 7 dias \\
\hline
\end{tabular}

${ }^{*} \mathrm{O}$ consumo de hidratos de carbono foi avaliado numa semana.

HC: Hidratos de Carbono

\section{Figura 2}

Meta-análise do consumo de hidratos de carbono durante o período competitivo

$\begin{array}{ll}\text { Estudos } & \text { Estimativa }(95 \% \text { IC) } \\ \text { Ruiz et al. 2005 } & 4,60(4,520 ; 4,680) \\ \text { Renon \& Collado (a) } 2013 & 3,60(3,258 ; 3,942) \\ \text { Renon \& Collado (b) } 2013 & 4,20(3,644 ; 4,756) \\ \text { Bettonviel et al. 2016 } & 4,70(4,333 ; 5,067) \\ \text { Andrews et al. (c) } 2016 & 3,50(3,004 ; 3,996) \\ \text { Andrews et al. (d) } 2016 & 3,90(3,266 ; 4,534) \\ \text { Anderson et al. (e) } 2017 & 4,20(3,080 ; 5,320) \\ \text { Anderson et al. (f) } 2017 & 6,40(4,640 ; 8,160) \\ \text { Brinkmans et al. (a) } 2019 & 3,90(4,441 ; 4,359) \\ \text { Brinkmans et al. (b) } 2019 & 5,10(4,522 ; 5,648) \\ & \\ \text { Geral: (I } \quad \text { (I) } 87,09 \%, p<0,001) & 4,268(3,903 ; 4,633)\end{array}$

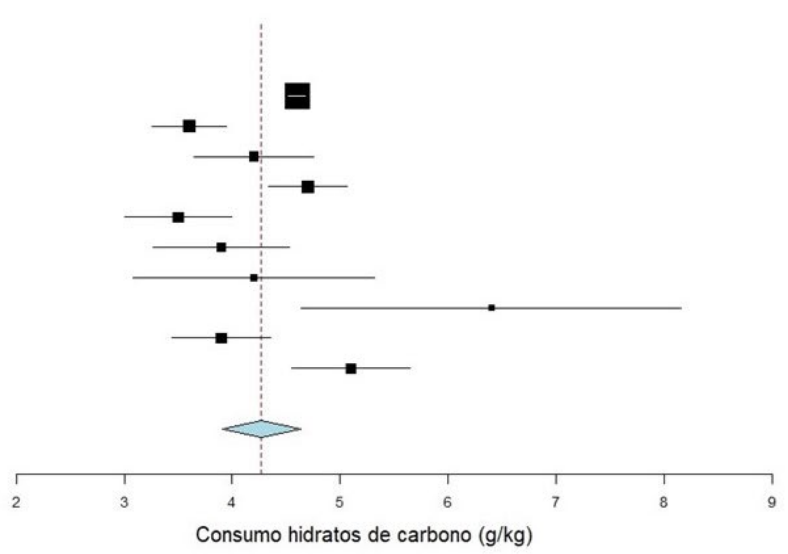

Consumo de Hidratos de Carbono Durante o Período Preparatório ou Pré-época

Quatro estudos foram incluídos na meta-análise, na qual foi estimado o consumo de hidratos de carbono em 5,1 g. $\mathrm{kg}^{-1} \cdot \mathrm{dia}^{-1}$ (95\% IC: 3,4 $\mathrm{g} \cdot \mathrm{kg}^{-1} \cdot \mathrm{dia}^{-1}$ a $6,6 \mathrm{~g} \cdot \mathrm{kg}^{-1}$.dia-1) durante a pré-epoca, em 63 futebolistas. O valor de heterogeneidade foi elevado ( $(=96 \%$; $p<0,001)$ - Figura 3

\section{Consumo de Hidratos de Carbono Durante a Off-season}

Nenhum estudo reportou o consumo de hidratos de carbono durante a off-season.

Variação no consumo de hidratos de carbono por grupo posicional A Tabela 2 sumaria o consumo de hidratos de carbono por grupo posicional. Na amostra que envolveu futebolistas do Brasil (24), o consumo de hidratos de carbono foi comparável nos diferentes grupos posicionais

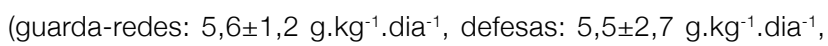

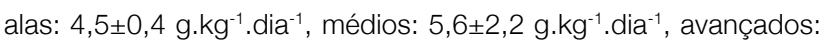
$\left.5,46 \pm 1,41 \mathrm{~g} \cdot \mathrm{kg}^{-1} \cdot \mathrm{dia}^{-1}\right)$, enquanto que na amostra dos Países Baixos (23) os guarda-redes $\left(3,6 \pm 0,8 \mathrm{~g} \cdot \mathrm{kg}^{-1} \cdot \mathrm{dia}^{-1}\right)$ e os avançados $\left(3,8 \pm 1,4 \mathrm{~g} \cdot \mathrm{kg}^{-1} \cdot \mathrm{dia}^{-1}\right)$ ingeriam menos hidratos de carbono do que os defesas $\left(4,2 \pm 1,4 \mathrm{~g} \cdot \mathrm{kg}^{-1} \cdot \mathrm{dia}^{-1}\right)$ e os médios $\left(4,0 \pm 0,8 \mathrm{~g} \cdot \mathrm{kg}^{-1} \cdot \mathrm{dia}^{-1}\right)$. 


$\begin{array}{ll}\text { Estudos } & \text { Esimativa }(95 \% \text { IC }) \\ \text { Noda et al. 2009 } & 6,90(6,055 ; 7,745) \\ \text { Devlin et al. 2016 } & 2,90(2,392 ; 3,408) \\ \text { Raizel et al. 2017 } & 5,40(4,546 ; 6,254) \\ \text { Kziazek et al. 2020 } & 5,10(4,639 ; 5,561) \\ \text { Geral: } \quad\left(I^{2}=96,22 \%, p<0,001\right) & 5,051(3,443 ; 6,659)\end{array}$

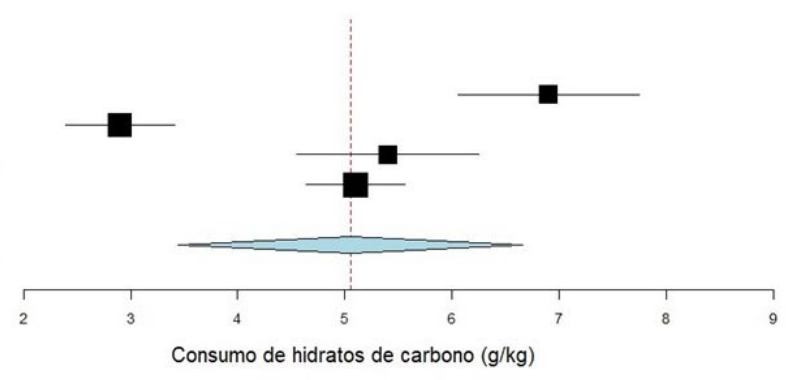

Tabela 2

Consumo de hidratos de carbono considerando a posição em campo

\begin{tabular}{|c|c|c|c|c|c|c|}
\hline ESTUDO & PAís & POSIÇÃo & $\begin{array}{c}\text { NÚMERO DE } \\
\text { PARTICIPANTES }\end{array}$ & IDADE & MÉDIA CONSUMO HC & $\begin{array}{l}\text { DESVIO PADRÃO } \\
\text { CONSUMO HC }\end{array}$ \\
\hline \multirow{5}{*}{ Raizel et al., 2017} & \multirow{5}{*}{ Brasil } & guarda-redes & 2 & 20,4 & 5,6 & 1,2 \\
\hline & & defesas & 4 & 19,0 & 5,5 & 2,7 \\
\hline & & alas & 2 & 21,0 & 4,5 & 0,4 \\
\hline & & médios & 8 & 20,4 & 5,6 & 2,2 \\
\hline & & avançados & 3 & 22,5 & 5,5 & 1,4 \\
\hline \multirow{4}{*}{ Brinkmans et al., 2019} & \multirow{4}{*}{ Países Baixos } & guarda-redes & 4 & 24 & 3,6 & 0,8 \\
\hline & & defesas & 12 & 25 & 4,2 & 1,4 \\
\hline & & médios & 13 & 22 & 4,0 & 0,8 \\
\hline & & avançados & 12 & 21 & 3,8 & 1,4 \\
\hline Anderson et al., 2018 & Inglaterra & guarda-redes & 1 & 26 & 2,6 & 0,6 \\
\hline
\end{tabular}

${ }^{1} \mathrm{O}$ consumo de hidratos de carbono foi estimado para um guarda-redes durante 7 dias. HC: Hidratos de carbono

Finalmente, o guarda-redes estudado em Inglaterra apresentou um consumo menor comparativamente aos guarda-redes avaliados no Brasil ou nos Países Baixos.

\section{DISCUSSÃO DOS RESULTADOS}

O objetivo desta revisão sistemática com meta-análise foi avaliar o consumo de hidratos de carbono em futebolistas, considerando a periodização anual da época. De acordo com recentes diretrizes publicadas por um grupo de peritos da UEFA (7), o consumo de hidratos de carbono durante a pré-epoca deve ser de 4-8 g. $\mathrm{kg}^{-1} \cdot \mathrm{dia}^{-1}$, enquanto na off-season a ingestão deve ser inferior a $4 \mathrm{~g} \cdot \mathrm{kg}^{-1} \cdot \mathrm{dia}^{-1}$. As recomendações para o período que embarga o calendário competitivo apresentam uma maior amplitude, variando entre 3-8 g. $\mathrm{kg}^{-1} \cdot \mathrm{dia}^{-1}$, nas semanas em que existe um jogo, e 6-8 g. $\mathrm{kg}^{-1} \cdot \mathrm{dia}^{-1}$ quando o calendário competitivo é mais congestionado. No entanto, mesmo para equipas que competem apenas uma vez por semana, é sugerido 6-8 g. $\mathrm{kg}^{-1} \cdot \mathrm{dia}^{-1}$ no dia antecedente e posterior ao jogo (7). Em média, os jogadores de futebol avaliados durante a pré-epoca, cumpriram as diretrizes de hidratos de carbono. Em contraste, durante a época, a ingestão de hidratos de carbono tende a estar próxima do limite inferior recomendado. Surpreendentemente, nenhum estudo avaliou a ingestão de hidratos de carbono durante a off-season.

A necessidade de periodizar o consumo de hidratos de carbono explica-se pelas exigências e objetivos específicos nas diferentes fases da época $(6,7)$. Durante o período preparatório ou pré-epoca, as diretrizes de hidratos de carbono justificam-se pela variabilidade da carga de treino imposta, enquanto, durante a fase competitiva, o objetivo é de sustentar a elevada frequência e intensidade de treino, para além de proporcionar aos atletas a reposição do glicogénio muscular e hepático (7). As diretrizes do consumo de hidratos do carbono durante o período competitivo foram definidas considerando o congestionamento do campeonato competitivo. Esta abordagem fundamentou-se num estudo recente que avaliou o impacto da carga de treino e de jogo em futebolistas de elite ingleses, durantes diferentes microciclos competitivos (26). Jogadores que competiram duas e três vezes, semanalmente, percorreram durante o treino e o jogo $32,5 \mathrm{~km}$ e $35,5 \mathrm{~km}$, respetivamente, enquanto os atletas que apenas tiveram um jogo completaram $25,9 \mathrm{~km}$. Adicionalmente, a percentagem de tempo em ações com velocidade acima de $14 \mathrm{~km} \cdot \mathrm{h}^{-1}$, foi superior quando os atletas competiam três vezes por semana (23\%) comparativamente a duas (18\%) ou uma vez (14\%) (26). Os dados previamente reportados têm implicações nas estratégias nutricionais a adotar nos diferentes microciclos da época competitiva. No entanto, o limite inferior de $3 \mathrm{~g} \cdot \mathrm{kg}^{-1}$. $\mathrm{dia}^{-1}$, na tentativa de otimizar o rendimento desportivo, parece não satisfazer as necessidades energéticas dos futebolistas.

O consumo de $3 \mathrm{~g} \cdot \mathrm{kg}^{-1} \cdot \mathrm{dia}^{-1}$ de hidratos de carbono, num futebolista de $80 \mathrm{~kg}$, traduz-se em $960 \mathrm{kcal}$ de hidratos de carbono. Se o mesmo atleta obedecer às recomendações proteicas e lipídicas de 1,6 g. $\mathrm{kg}^{-1}$ . dia ${ }^{-1}$ e 1,2 g. $\mathrm{kg}^{-1}$. dia-1 sugeridas num estudo com 41 futebolistas de elite (23), respetivamente, o seu consumo energético será aproximadamente de $2400 \mathrm{kcal}$ por dia. Este valor é inferior ao gasto energético estimado por água duplamente marcada em futebolistas de elite ingleses (10) e holandeses (23), os quais despendem, em média, $3186 \mathrm{kcal}$ e $3566 \mathrm{kcal}$, respetivamente. Extrapolando os resultados encontrados no presente estudo, no qual a média do consumo de hidratos de carbono, durante o período competitivo foi de $4,3 \mathrm{~g} \cdot \mathrm{kg}^{-1} \cdot \mathrm{dia}^{-1}$, um atleta de $80 \mathrm{~kg}$ ingeriria aproximadamente, 2500 kcal, o que não permitiria satisfazer o seu dispêndio energético. O consumo de proteína anteriormente mencionado, 1,6 g. $\mathrm{kg}^{-1} \cdot \mathrm{dia}^{-1}$, 
em 41 futebolistas dos Países Baixos foi consideravelmente inferior aquele que foi reportado em futebolistas Britânicos $\left(2,4 \mathrm{~g} \cdot \mathrm{kg}^{-1}\right)$, o que demonstra a necessidade de estudos futuros descreverem o consumo nutricional em futebolistas profissionais (27).

A amplitude do consumo de hidratos de carbono já havia sido recentemente discutida, sendo sugeridos $7-8 \mathrm{~g} \cdot \mathrm{kg}^{-1} \cdot \mathrm{dia}^{-1} \mathrm{em}$ períodos de treino intensos ou momentos competitivos (23). O presente estudo parece sugerir um estreitamento das diretrizes do consumo de hidratos de carbono no período competitivo, independentemente, do congestionamento do calendário. Mesmo em períodos da época, em que só existe um momento competitivo por semana, as 24-36 horas anteriores representam um período temporal que justifica um aporte superior de hidratos de carbono, conjugado com unidades de treino de baixa intensidade (6). A ingestão de $4,3 \mathrm{~g} \cdot \mathrm{kg}^{-1} \cdot \mathrm{dia}^{-1} \mathrm{de}$ hidratos de carbono, durante o período competitivo pode ser explicada por um consumo excessivo de proteína $(10,17,20,22,24)$. Outra explicação para os valores estimados de hidratos de carbono poderá ser a metodologia usada para avaliar o seu consumo. A maioria dos estudos estimaram a ingestão de hidratos de carbono recorrendo ao recordatório das 24 horas anteriores ou ao diário alimentar. Note-se, que os erros associados aos diários alimentares, tendem a subestimar 18-20\% o consumo energético real (5). Para minimizar o erro da estimativa da ingestão de hidratos de carbono (28), os estudos que avaliaram futebolistas de elite ingleses recorreram ao diário alimentar de 7 dias, combinado com o recordatório das 24 horas anteriores. Outro fator que pode influenciar os valores encontrados no consumo de hidratos de carbono é a variação por posição. Um estudo com 19 futebolistas brasileiros reportou ingestões comparáveis entre os diferentes grupos posicionais (24), contrastando com os resultados verificados em 41 futebolistas holandeses, com os guarda-redes a reportarem um consumo inferior comparativamente aos defesas, médios e avançados (23). Um guarda-redes inglês avaliado durante sete dias reportou um consumo diário inferior a $3 \mathrm{~g} \mathrm{~kg}^{-1}$. dia-1 , com um dispêndio energético médio de 3160 kcal (19), substancialmente inferior às $3600 \mathrm{kcal}$ estimadas em 6 futebolistas ingleses de outras posições em campo.

A presente revisão sistemática e meta-análise tem limitações que devem ser reconhecidas. Primeiramente, o consumo de hidratos de carbono nos dias de treino e competição foram considerados para avaliar a ingestão durante o período competitivo, pelo que estudos futuros devem clarificar se o consumo de hidratos de carbono deve ser distinto nos dias de treino e competição, comparando com o dispêndio energético e a variabilidade da carga. Finalmente, os resultados da meta-análise devem ser cuidadosamente interpretados tendo em conta o nível de heterogeneidade encontrada.

\section{CONCLUSÕES}

Em suma, o consumo de hidratos de carbono em jogadores de futebol treinados aproxima-se do limite inferior recomendado considerando as mais recentes diretrizes durante o período competitivo. Durante o período preparatório cumprem, em média, as recomendações sugeridas. Apesar da frequente atualização das diretrizes para o consumo de hidratos de carbono $(7,29)$, o presente estudo evidenciou que são necessárias estratégias para que os atletas percebam a necessidade deste macronutriente durante o período competitivo, considerando também as suas preferências alimentares (20). A variabilidade por posição deve ser considerada aquando da ingestão alimentar, emergindo do presente estudo que os guarda-redes apresentam um consumo de hidratos de carbono inferior ao de atletas das restantes posições em campo.

\section{REFERÊNCIAS BIBLIOGRÁFICAS}

1. Krustrup P, Mohr M, Steensberg A, Bencke J, Kjaer M, Bangsbo J. Muscle and blood metabolites during a soccer game: implications for sprint performance. Med Sci Sports Exerc. 2006;38(6):1165-74.

2. Bangsbo J, Mohr M, Krustrup P. Physical and metabolic demands of training and match-play in the elite football player. J Sports Sci. 2006;24(7):665-74.

3. Bangsbo J, laia FM, Krustrup P. Metabolic response and fatigue in soccer. Int $J$ Sports Physiol Perform. 2007;2(2):111-27.

4. Balsom PD, Wood K, Olsson P, Ekblom B. Carbohydrate intake and multiple sprint sports: with special reference to football (soccer). Int J Sports Med. 1999;20(1):48-52. 5. Burke LM, Cox GR, Culmmings NK, Desbrow B. Guidelines for daily carbohydrate intake: do athletes achieve them? Sports Med. 2001;31(4):267-99.

6. Burke LM, Loucks AB, Broad N. Energy and carbohydrate for training and recovery. J Sports Sci. 2006;24(7):675-85.

7. Collins J, Maughan RJ, Gleeson M, Bilsborough J, Jeukendrup A, Morton JP, Phillips SM, Armstrong L, Burke LM, Close GL, Duffield R, Larson-Meyer E, Louis J, Medina D, Meyer F, Rollo I, Sundgot-Borgen J, Wall BT, Boullosa B, Dupont G, Lizarraga A, Res P, Bizzini M, Castagna C, Cowie CM, D'Hooghe M, Geyer H, Meyer T, Papadimitriou $\mathrm{N}$, Vouillamoz M, McCall A. UEFA expert group statement on nutrition in elite football. Current evidence to inform practical recommendations and guide future research. $\mathrm{Br}$ J Sports Med. 2021;55(8):416.

8. Malone JJ, Di Michele R, Morgans R, Burgess D, Morton JP, Drust B. Seasonal training-load quantification in elite English premier league soccer players. Int J Sports Physiol Perform. 2015;10(4):489-97.

9. Boone J, Vaeyens R, Steyaert A, Vanden Bossche L, Bourgois J. Physical fitness of elite Belgian soccer players by player position. J Strength Cond Res. 2012;26(8):2051-7. 10. Anderson L, Close GL, Morgans R, Hambly C, Speakman JR, Drust B, Morton JP. Assessment of Energy Expenditure of a Professional Goalkeeper From the English Premier League Using the Doubly Labeled Water Method. Int J Sports Physiol Perform. 2019 1;14(5):681-684.

11. Anderson L, Orme P, Naughton RJ, Close GL, Milsom J, Rydings D, O'Boyle A, Di Michele R, Louis J, Hambly C, Speakman JR, Morgans R, Drust B, Morton JP. Energy Intake and Expenditure of Professional Soccer Players of the English Premier League: Evidence of Carbohydrate Periodization. Int J Sport Nutr Exerc Metab. 2017;27(3):228-238. 12. Williams C, Rollo I. Carbohydrate Nutrition and Team Sport Performance. Sports Med. 2015;45 Suppl 1(Suppl 1):S13-22.

13. Liberati A, Altman DG, Tetzlaff J, Mulrow C, Gøtzsche PC, loannidis JP, Clarke M, Devereaux PJ, Kleijnen J, Moher D. The PRISMA statement for reporting systematic reviews and meta-analyses of studies that evaluate health care interventions: explanation and elaboration. J Clin Epidemiol. 2009;62(10):e1-34.

14. Kohl C, Mclntosh EJ, Unger S, Haddaway NR, Kecke S, Schiemann J, Wilhelm R. Online tools supporting the conduct and reporting of systematic reviews and systematic maps: a case study on CADIMA and review of existing tools. Environ Evid. 2018, 7(1), 1-17. 15. Moola S, Munn Z, Tufanaru C, Aromataris E, Sears K, Sfetcu R, Currie M, Lisy K, Qureshi R, Mattis P, Mu P. Chapter 7: Systematic reviews of etiology and risk. In: Aromataris E, Munn Z (Editors). JBI Manual for Evidence Synthesis. JBI, 2020. Available from https://synthesismanual.jbi.global. https://doi.org/10.46658/JBIMES-20-08.

16. Higgins JP, Thompson SG. Quantifying heterogeneity in a meta-analysis. Stat Med. 2002;21(11):1539-58.

17. Ruiz F, Irazusta A, Gil S, Irazusta J, Casis L, Gil J. Nutritional intake in soccer players of different ages. J Sports Sci. 2005 Mar;23(3):235-42.

18. Martínez Reñón C, Sánchez Collado P. Estudio nutricional de un equipo de fútbol de tercera división [Nutritional study of a third division soccer team]. Nutr Hosp. 2013;28(2):319-24

19. Noda Y, lide K, Masuda R, Kishida R, Nagata A, Hirakawa F, Yoshimura Y, Imamura $\mathrm{H}$. Nutrient intake and blood iron status of male collegiate soccer players. Asia Pac $J$ Clin Nutr. 2009;18(3):344-50.

20. Devlin BL, Leveritt MD, Kingsley M, Belski R. Dietary Intake, Body Composition, and Nutrition Knowledge of Australian Football and Soccer Players: Implications for Sports Nutrition Professionals in Practice. Int J Sport Nutr Exerc Metab. 2017;27(2):130-138. 
21. Andrews MC, Itsiopoulos C. Room for Improvement in Nutrition Knowledge and Dietary Intake of Male Football (Soccer) Players in Australia. Int J Sport Nutr Exerc Metab. 2016;26(1):55-64.

22. Bettonviel A EO, Brinkmans N YJ, Russcher K, Wardenaar FC, Witard OC. Nutritional Status and Daytime Pattern of Protein Intake on Match, Post-Match, Rest and Training Days in Senior Professional and Youth Elite Soccer Players. Int J Sport Nutr Exerc Metab. 2016;26(3):285-93.

23. Brinkmans NYJ, ledema N, Plasqui G, Wouters L, Saris WHM, van Loon LJC, van Dijk JW. Energy expenditure and dietary intake in professional football players in the Dutch Premier League: Implications for nutritional counselling. J Sports Sci. 2019;37(24):2759-2767.

24. Raizel R, da Mata Godois A, Coqueiro AY, Voltarelli FA, Fett CA, Tirapegui J, de Paula Ravagnani FC, de Faria Coelho-Ravagnani C. Pre-season dietary intake of professional soccer players. Nutr Health. 2017;23(4):215-222.

25. Książek A, Zagrodna A, Słowińska-Lisowska M. Assessment of the Dietary Intake of High-Rank Professional Male Football Players during a Preseason Training Week. Int J Environ Res Public Health. 2020;17(22):8567.

26. Anderson L, Orme P, Di Michele R, Close GL, Morgans R, Drust B, Morton JP. Quantification of training load during one-, two- and three-game week schedules in professional soccer players from the English Premier League: implications for carbohydrate periodisation. J Sports Sci. 2016;34(13):1250-9.

27. Hulton AT, Malone JJ, Clarke ND, MacLaren DPM. Energy Requirements and Nutritional Strategies for Male Soccer Players: A Review and Suggestions for Practice. Nutrients. 2022; 14(3):657.

28. Braakhuis AJ, Meredith K, Cox GR, Hopkins WG, Burke LM. Variability in estimation of self-reported dietary intake data from elite athletes resulting from coding by different sports dietitians. Int J Sport Nutr Exerc Metab. 2003;13(2):152-65.

29. Fernandes HS. Carbohydrate Consumption and Periodization Strategies Applied to Elite Soccer Players. Curr Nutr Rep. 2020;9(4):414-419. 